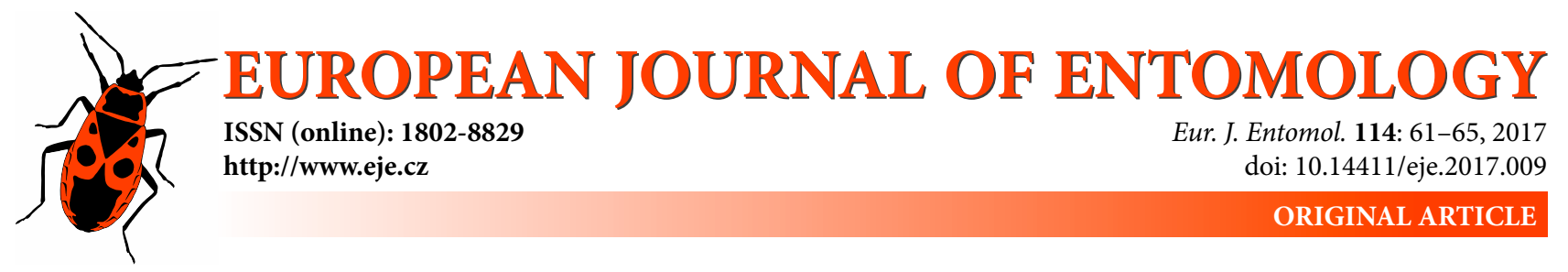

\title{
Silk recycling in larvae of the wax moth, Galleria mellonella (Lepidoptera: Pyralidae)
}

\author{
HaQ Abdul ShalK ${ }^{1,2}$, Archana MiShRA ${ }^{1}$ and František SeHNAL ${ }^{1,2}$ \\ ${ }^{1}$ Biology Centre ASCR, Institute of Entomology, Branišovská 31, 37005 České Budějovice, Czech Republic; \\ e-mails: haq@entu.cas.cz, archanaphd@gmail.com, sehnal@bc.cas.cz \\ 2 University of South Bohemia, Faculty of Science, Branišovská 31, 37005 České Budějovice, Czech Republic
}

Key words. Lepidoptera, Pyralidae, silk recycling, larval silk, cocoon silk, Galleria mellonella, Bombyx mori

\begin{abstract}
Galleria mellonella larvae spin protective tubes, which they use until they finish feeding, when they spin cocoons. A feeding choice experiment showed that some of the silk produced by feeding larvae was consumed in addition to the standard diet (STD). To determine the effect of feeding on silk, last instar larvae were fed for $24 \mathrm{~h}$ on foods based on STD but modified by replacing the dry milk component ( $10 \%$ of the diet) with equal amounts of different kinds of silk. While each control larva consumed $21 \pm 0.5 \mathrm{mg}$ of the STD and produced $4.5 \pm 0.1 \mathrm{mg}$ of silk, larvae that ate the food that contained larval silk consumed $10 \pm 0.4 \mathrm{mg}$ of food and produced $6.1 \pm 0.1 \mathrm{mg}$ silk; the percentage ratio of silk produced to diet consumed was $21 \%$ and $61 \%$, respectively. A more pronounced reduction in food consumption occurred when larvae were supplied with Galleria "cocoon" silk or the sericin fraction of such silk, and only $3.8 \mathrm{mg} /$ larva was ingested of the diet containing Bombyx mori cocoon silk or its sericin fraction. Silk production expressed in terms of percentage of diet consumed was always higher than that recorded for larvae fed STD. We conclude that G. mellonella larvae recycle part of the silk that they produce during feeding. Presence of silk in the diet reduces food intake but increases the ratio of silk production to diet consumption. Sericin fraction of the cocoon silk seems to deter feeding.
\end{abstract}

Abbrevations. BCF - Bombyx cocoon fibroin, BCS - Bombyx cocoon silk, BPS - Bombyx pad silk, BS - Bombyx sericin, CBS - Commercial Bombyx sericin, GCF - Galleria cocoon fibroin, GCS - Galleria cocoon silk, GLS - Galleria larval silk, GS Galleria sericin, STD - Standard diet.

\section{INTRODUCTION}

Silk produced by the larvae of several holometabolous insect orders is typically used for the construction of cocoons at the end of larval development but in a number of cases is also used for other purposes, including protection of feeding larvae (Sutherland et al., 2010). The caterpillars of the wax moth, Galleria mellonella (Lepidoptera: Pyralidae), living in beehives, spin protective tubes in which they are not detected by the bees. Since the tube is continuously expanded as the larva grows, the amount of protein invested in the silk during larval development is considerable. The last larval instar alone adds about four times more silk to the larval tube than it invests in the cocoon it spins after it ceases feeding (Jindra \& Sehnal, 1989).

The composition of "larval silk" used in the construction of the tubes is similar to the "cocoon silk". The core of the silk filament is made up of heavy and light chain fibroins and the P25 chaperonin, and the filament coating is composed of sericins (Fedič et al., 2002). The sericin genes $M G-1, M G-2$ and $M G-3$, which are differentially expressed and spliced in the course of larval development and prior to cocoon spinning, produce at least 7 different proteins in G. mellonella (Žurovec et al., 2013). There are also pronounced developmental differences in the expression of the minor silk components, the seroins (Žurovec et al., 1998) and the proteinase inhibitors (Nirmala et al., 2001a,b): their genes are silent in the silk glands of feeding larvae and become highly active at the onset of cocoon spinning. Hence, the "larval" and "cocoon" silks are not identical but the significance of the differences is unknown. In the present study we demonstrate differences between the consumption of larval and cocoon silk. We suggest that the ingestion of larval silk enables larvae to recycle this silk, but a feeding deterrent inhibits the destruction of the more durable cocoon silk.

In nature, G. mellonella larvae feed on honeycombs that contain a large proportion of beeswax, some honey, cast exuviae of immature bee stages, and pollen residues. Larvae obtain a large amount of energy from such food but little protein. Under laboratory conditions the larvae develop successfully on artificial diets of different compositions (Dadd, 1964). However, if the amount of protein in the diet drops below a certain threshold, the larvae stop spinning (Jindra \& Sehnal, 1989), presumably due to the shortage of essential amino acids needed for the synthesis of silk 
proteins. For the silk producing larvae of G. mellonella it would be clearly advantageous to consume silk of the abandoned tubes and recycle the amino acids.

Several aspects of the relationship between diet and silk production are reported by Craig et al. (1999) who compared amino acid compositions of silks produced by herbivores and carnivores. Craig et al. (2000) subsequently confirmed the influence of diet on silk production by spiders [see Tso et al. (2005) and Blamires et al. $(2012,2015)$ for further information]. Comparable data are not available for Lepidoptera that are the most prolific silk-spinning arthropods next to spiders. The caterpillars of $B$. mori produce and store silk in a pair of silk glands that make up to $60 \%$ of their body mass at the beginning of cocoon spinning (Prudhomme et al., 1985).

This study addresses the possibility of silk recycling in G. mellonella along with another intriguing question about the dietary properties of silk. It is proposed that minor silk components such as seroins and proteinase inhibitors protect cocoons against microorganisms and possibly also against mites, insects and other omnivorous animals (Nirmala et al., 2001a, b; Shaik \& Sehnal, 2009; Singh et al., 2014). To answer both questions, we fed larvae with raw or processed silk and determined their feeding rate and silk production.

\section{MATERIALS AND METHODS}

\section{Insect rearing}

Larvae of the wax moth, G. mellonella (Lepidoptera: Pyralidae), were obtained from a laboratory stock culture fed a standard diet at $30^{\circ} \mathrm{C}$ in total darkness (Jindra \& Sehnal, 1989). Under these conditions, the whole life cycle was completed in about 40 days. Within 14-20 days after hatching, the larvae underwent six ecdyses and reached the final (seventh) larval instar, which included a 5-day feeding period followed by wandering and cocoon spinning. The larvae ecdysed to pupae 2-3 days after they ceased feeding. In our feeding assays we used larvae 2 days after the last larval ecdysis (body weight $80-120 \mathrm{mg}$ ). This stage was chosen because it was the time of voracious feeding and intensive construction of the protective silk tubes.

\section{Diets tested}

The standard diet (STD) was prepared from components preheated to $80^{\circ} \mathrm{C}$. Dry ingredients $(100 \mathrm{mg}$ wheat flour, $200 \mathrm{mg}$ maize bran, $100 \mathrm{mg}$ wheat meal, $100 \mathrm{mg}$ dry milk and $50 \mathrm{mg}$ dry yeast) were consecutively kneaded with $150 \mathrm{mg}$ beeswax, 100 mg glycerin and eventually with $200 \mathrm{mg}$ honey (Jindra \& Sehnal, 1989). Silk diets were prepared by replacing dry milk with an equal amount (by weight) of silk or a silk component obtained from G. mellonella and Bombyx mori, respectively. The diets were named after the silk component: Galleria larval silk (GLS), Galleria cocoon silk (GCS), Bombyx pad silk (BPS), Bombyx cocoon silk (BCS), Galleria cocoon fibroin (GCF), Bombyx cocoon fibroin (BCF), Galleria sericin (GS), Bombyx sericin (BS) and Commercial Bombyx sericin (CBS). Clean silk samples were cut into tiny pieces and thoroughly mixed with the dry diet components. The fibroin and sericin silk fractions were prepared from $0.5 \mathrm{~g}$ samples of chopped cocoons that were ground to powder in liquid nitrogen. The powder was suspended in $7.5 \mathrm{ml}$ of $5 \mathrm{mM}$ sodium phosphate buffer ( $\mathrm{pH} \mathrm{7.0)}$ and incubated at $99^{\circ} \mathrm{C}$ for $60 \mathrm{~min}$. The supernatant (solubilized fraction), which is the sericin extract, was separated from the insoluble fibroin fraction by centrif-

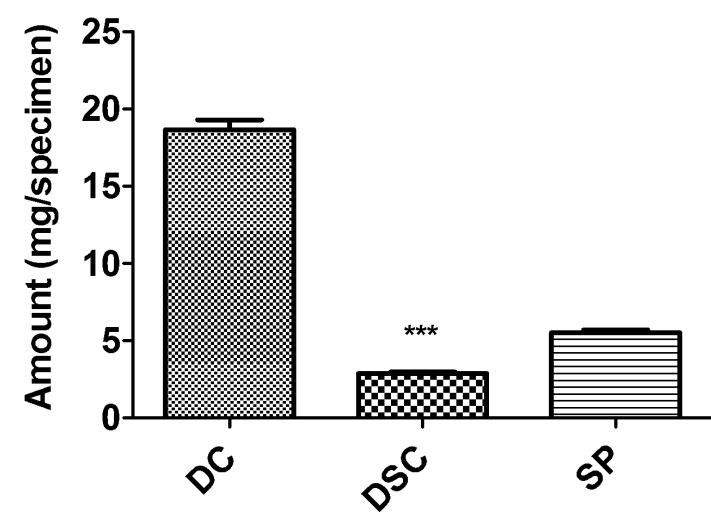

Fig. 1. Consumption of the standard diet (DC) and dyed larval silk (DSC), and production of new silk (SP) over a period of $24 \mathrm{~h}$ by last instar larvae of $G$. mellonella (Means \pm SEM values per specimen). That the difference between dyed silk consumption and new silk production is significant based on a Student's t-test is indicated by asterisks $(n=6)$.

ugation at $4000 \mathrm{~g}$ for $90 \mathrm{~min}$. Both fractions were vacuum dried, weighed and aliquots of them were used in the GCF, BCF, GS and BS diets. Commercial hydrolysate of B. mori sericins (WAKO Co., Japan) was tested in the CBS diet. Galleria larval silk stained with a culinary dye so that it could be recognized from the newly produced silk was offered to larvae as an alternative to STD in the feeding choice experiment.

\section{Feeding assays}

A feeding choice experiment was done to check if larvae consume silk when also offered the STD. Three sets of eight Petri dishes (18 $\mathrm{cm}$ in diameter), each with two larvae, were provided with weighed amounts of STD and of Galleria larval silk (GLS) stained with a culinary dye. After $24 \mathrm{~h}$ we measured the consumption of STD and of the dyed silk, and simultaneously also the amount of silk produced during the experiment.

Possible effects of silks on larval feeding and silk production were determined in no-choice experiments comparing the performance of larvae fed on the diets described above. Two larvae were placed in each Petri dish ( $18 \mathrm{~cm}$ diameter) and supplied with pre-weighed amounts (about $80 \mathrm{mg}$ per dish) of food. The food consumption and silk production were measured after $3,6,18$, and $24 \mathrm{~h}$. The experiment was repeated six times.

\section{Statistical analysis}

All data are presented as Mean \pm SEM. Statistical verification of differences between compared groups was done using Student's t-test (Fig. 1) and One-way analysis of variance (ANOVA) followed by Tukey's Multiple Comparison test (Fig. 4) in GraphPad Prism (Version 4.5, GraphPad Software, Inc., USA). Twoway ANOVA followed by the Holm-Sidak method of all-pairwise multiple comparison (Figs 2 and 3) was done in SigmaPlot (Version 11, SPSS Science, USA).

\section{RESULTS AND DISCUSSION}

In the feeding choice experiment, the consumption of STD during $24 \mathrm{~h}$ amounted to $19 \pm 0.6 \mathrm{mg}$ per larva and simultaneous ingestion of the dyed silk to $2.9 \pm 0.1 \mathrm{mg}$. In the same time interval, each larva produced an average of $5.5 \pm 0.2 \mathrm{mg}$ of new silk (Fig. 1). It is obvious that larvae consumed the silk deliberately because they could feed ad libitum on STD. Student's t-test confirmed that silk consumption was lower than silk production $(\mathrm{P}<0.05)$ but ingested silk could nevertheless provide nutrients for about 


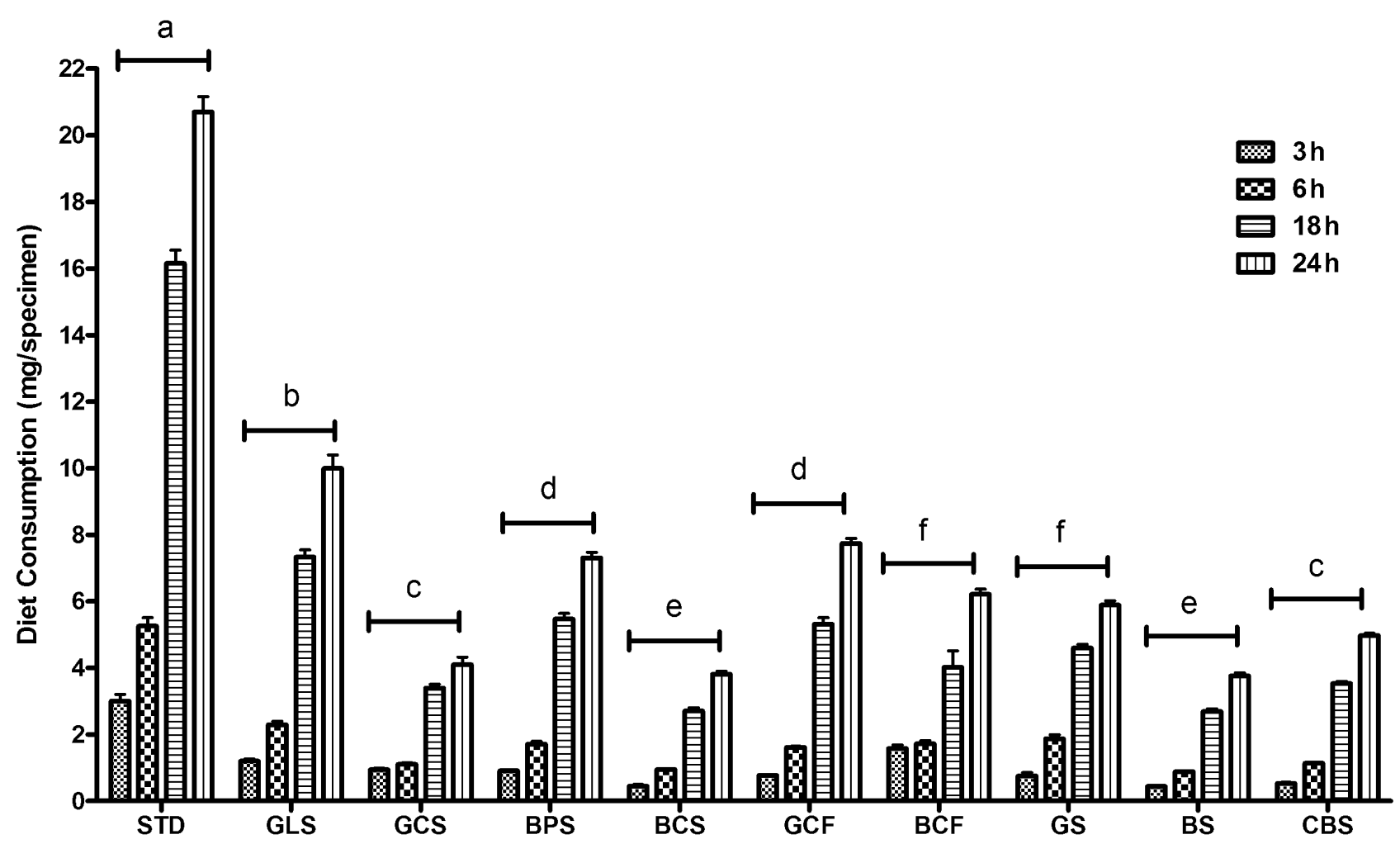

Fig. 2. Diet consumption (mg/specimen) by larvae of G. mellonella fed on the standard diet (STD) or on the modified diets (see Material and Methods) for 3, 6, 18 and $24 \mathrm{~h}$. Data are presented as Means \pm SEM. Two way ANOVA $(P<0.05)$ with the Holm-Sidak method was applied for an all-pairwise multiple comparison $(n=6)$. Different letters indicate significant differences.

half of the new silk. The recycling of the essential amino acids is probably particularly important for $G$. mellonella. While in B. mori the heavy chain fibroin (the major silk protein) contains $8.4 \%$ essential amino acids, that of $G$. mellonella contains $18.4 \%$ (Žurovec \& Sehnal, 2002). The benefits of silk recycling are demonstrated in spiders. For example, Opell (1998) report that the total cost of producing an orb-web was reduced by $32 \%$ when a spider recycles its silk. It is obvious that the production of silks composed of amino acids that are costly to synthesize is likely to place a greater drain on an animal's energy resources than silks composed of amino acids that are metabolically simple to produce or ingested directly from the diet or recycled (Craig et al., 1999).

Substituting dry milk with silk or a silk component had profound effects on diet consumption and silk spinning in G. mellonella. The control larvae consumed $21.0 \pm 0.5 \mathrm{mg}$ STD per larva, whereas larvae fed GLS consumed only $10 \pm 0.4 \mathrm{mg}$ (Fig. 2). The consumption of food with other silk components was still lower. Minimum ingestion (3.8 \pm $0.1 \mathrm{mg} /$ larva) was recorded for larvae fed the BS and BCS diets. The replacement of dry milk with the Bombyx cocoon silk (BCS) or with the sericin extract from such silk (BS), reduced diet consumption about 5.5 times. Only a slightly (but significantly) lower effect on diet consumption was recorded when dry milk was replaced by commercial $B$. mori sericin (CBS) or with Galleria cocoon silk (GCS). The other silk components tested were less effective but also significantly reduced food intake compared to STD. Two-way ANOVA of the experimental series with different diets revealed significant differences in the consumption of the different diets $(\mathrm{F}=948.97, \mathrm{P}<0.001)$ as well as differences in the amounts consumed within 3, 6, 18 and $24 \mathrm{~h}(\mathrm{~F}=2858.36, \mathrm{P}<0.001)$. All of the pairwise multiple comparisons of the effect of different diets revealed significant differences at $\mathrm{P}<0.001$, except for GCS vs BCS with $\mathrm{P}<0.05$. No difference was found between CBS vs GCS, BCF vs GS, BCS vs BS and GCF vs BPS. The all-pairwise multiple comparison of the amount of diet consumed in 3 , 6, 18 and $24 \mathrm{~h}$ long experiments verified that it increased over time $(\mathrm{P}<0.001)$.

The consumption of GLS was less than a half of the STD uptake but it was still nearly 2.5 times higher than the consumption of GCS (Fig. 2). It is possible that cocoon silk differs from larval silk in the content of compounds possessing feeding-deterrent properties. To get some insight into their localization, we tested separately the fibroin and sericin fractions of cocoon silk. For both G. mellonella and $B$. mori cocoons we found that the diets with fibroin fractions were consumed more readily than those with sericin fractions, indicating that the components that reduce feeding are in the silk fiber coating rather than in the fibroin core. This is consistent with the reports that the genes encoding compounds believed to provide cocoon protection against omnivores are expressed mainly or exclusively in the middle silk gland shortly before cocoon spinning (Žurovec et al., 1998; Nirmala et al., 2001a, b).

The rate of silk production (Fig. 3) to some extent reflected the rate of food consumption and its dependence on the diet. Our statistical analysis revealed a significant 


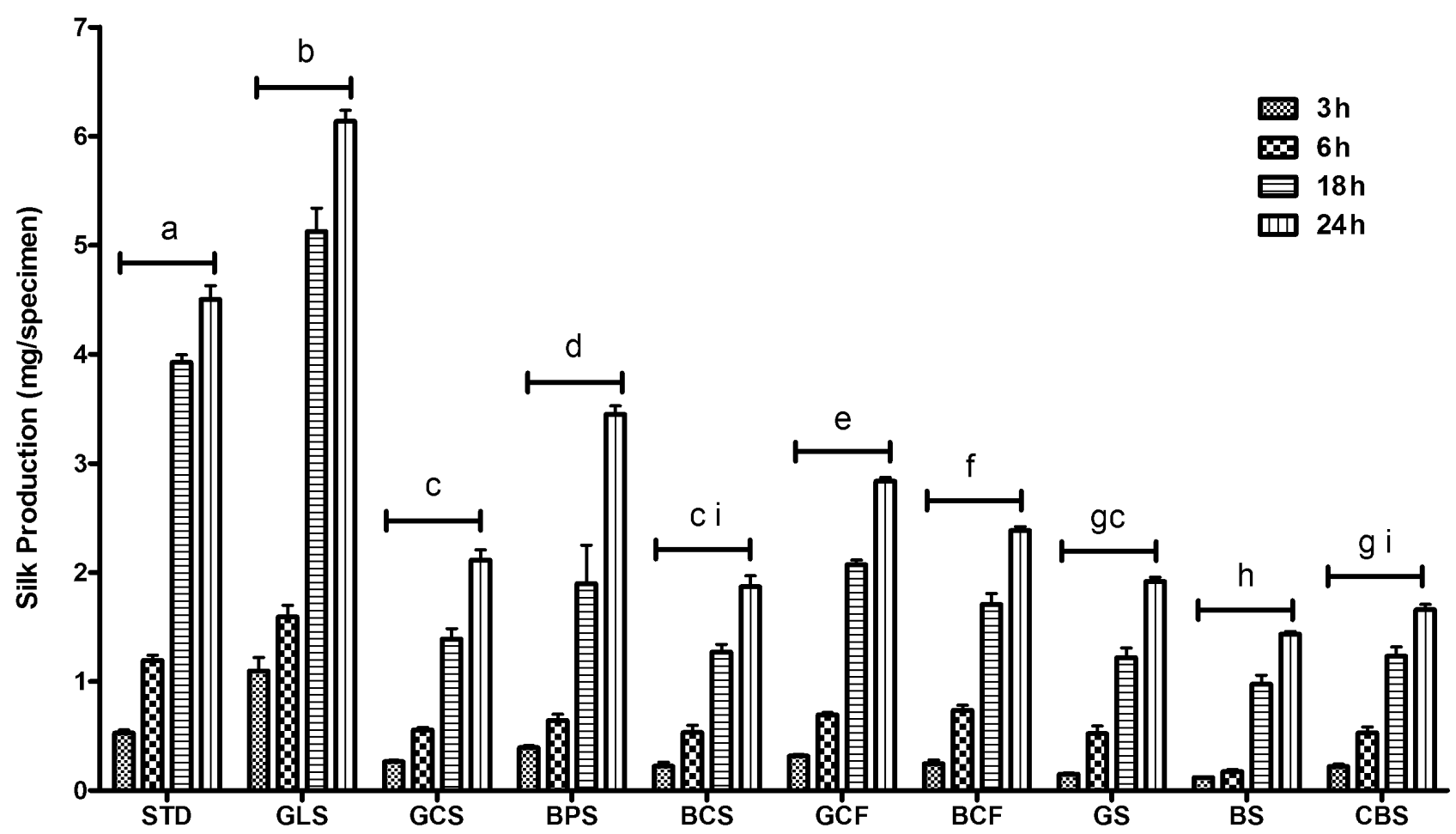

Fig. 3. Silk production (mg/specimen) by larvae of G. mellonella fed on STD (control) or on the modified diets (see Material and Methods) for 3, 6, 18 and $24 \mathrm{~h}$. Data are presented as Means \pm SEM. Two way ANOVA $(P<0.05)$ with the Holm-Sidak method was applied for an all-pairwise multiple comparison $(n=6)$. Different letters indicate significant differences.

effect of the type of diet $(\mathrm{F}=656.79, \mathrm{P}<0.001)$ and time $(\mathrm{F}=2631.10, \mathrm{P}<0.001)$ on the silk production. All pairwise multiple comparisons of the type of diet, and time were significant $(\mathrm{P}<0.001)$, except for GCS vs GS, BCS vs $\mathrm{CBS}$, GS vs CBS and BCS vs GS, which were not significant. Maximum silk production was recorded for GLS $(6.1 \pm 0.1)$ followed by STD $(4.5 \pm 0.1)$. Lowest silk output was recorded for larvae $(1.4 \pm 0.0)$ fed the BS diet (Fig. 3).

GLS proved to be the best diet for stimulating silk production (Fig. 3). The consumption of GLS was less than half of the STD uptake, but silk production was higher on GLS. For larvae feeding on STD, the amount of silk secret-

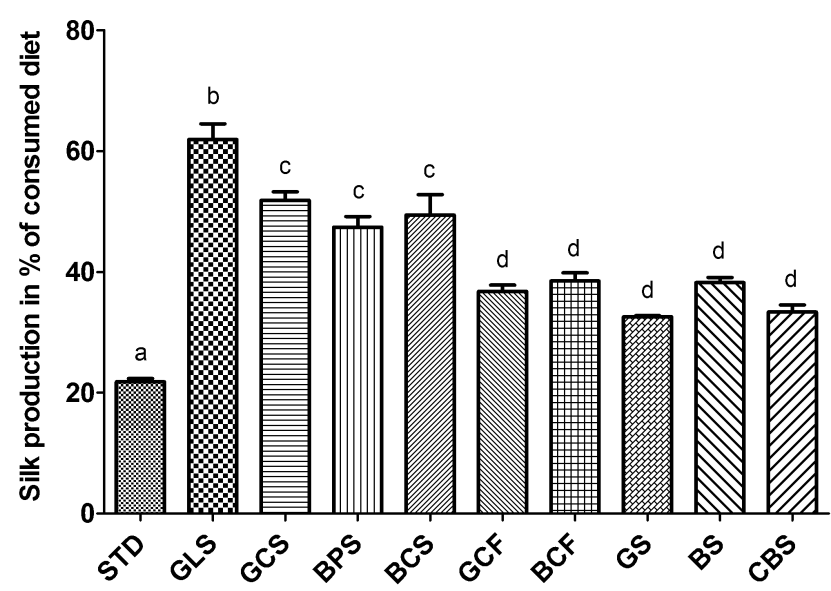

Fig. 4. Silk production expressed as a percentage of the food consumed by larvae fed on STD (control) or one of the modified diets (see Material and Methods). Data were evaluated using One-way ANOVA with Tukey's Multiple Comparison Test $(n=6-12)$. Different letters indicate significant differences. ed corresponded to $21 \%$ of the diet consumed, while on the GLS diet it was $61 \%$; hence, the ratio of silk production to the food uptake was about 3 times higher on the GLS diet. The ratio between silk produced and food consumed was significantly lower for larvae fed STD than larvae fed any of the silk-containing diets (Fig. 4). This is the most surprising finding of this study. It indicates that the nutrients needed for silk production are more abundant or accessible in the diets with silks than in those with dry milk.

In summary, we show that G. mellonella larvae consume some of the silk that they produce during their feeding period. However, the replacement of dry milk by silk in their diet resulted in a reduction in food consumption, possibly due to inferior silk digestibility. In spite of the lower food intake, the silk production was higher on a diet with silk than on the standard diet with dry milk. High silk production at a low food consumption suggests that nutrients from the digested silk are rapidly recycled. Our tentative explanation is that silk digestion slows down feeding but the silk digested is preferentially used for the synthesis of new silk. These interactions are probably complicated by the presence of compounds that have direct gustatory effects. Comparison of the effects of different silk materials on food consumption indicates the presence of a feeding deterrent in the sericin fraction of cocoon silk. This may be the major mechanism by which cocoons are protected against potential silk consumers and insectivores.

ACKNOWLEDGEMENTS. This study was done in the Entomological Institute, Biology Centre CAS, within the research program Z50070568. H.A. Shaik was supported by grant ME 907 from the Ministry of Education, Youth and Sports, Czech Repub- 
lic. We appreciate advice provided by our colleague Z. Svobodová on the use of statistics. We are also grateful to N. Krishnan, Mississippi State University and D. Kodrik, Biology Centre CAS for their advice and comments that greatly improved this manuscript.

\section{REFERENCES}

Blamires S.J., Wu C.L. \& Tso I.M. 2012: Variations in protein intake induces variations in spider silk expression. - PLOS ONE 7(2): e31626, 9 pp.

Blamires S.J., Liao C.P., Chang C.K., Chuang Y.C., Wu C.L., Blackledge T.A., Sheu H.S. \& Tso I.M. 2015: Mechanical performance of spider silk is robust to nutrient-mediated changes in protein composition. - Biomacromolecules 16: 1218-1225.

Craig C.L., Hsu M., Kaplan D. \& Pierce N.E. 1999: A comparison of the composition of silk proteins produced by spiders and insects. - Int. J. Biol. Macromol. 24: 109-118.

Craig C.L., Riekel C., Herberstein M.E., Weber R.S., Kaplan D. \& PIERCE N.E. 2000: Evidence for diet effects on the composition of silk proteins produced by spiders. - Mol. Biol. Evol. 17: 1904-1913.

DADD R.H. 1964: A study of carbohydrate and lipid nutrition in the wax moth, Galleria mellonella (L.), using partially synthetic diets. - J. Insect Physiol. 10: 161-178.

Fedič R., Žurovec M. \& SeHnal F. 2002: The silk of Lepidoptera. - J. Insect Biotechnol. Sericol. 71: 1-15.

JINDRA M. \& SeHNaL F. 1989: Larval growth, food consumption, and utilization of dietary protein and energy in Galleria mellonella. - J. Insect Physiol. 35: 719-724.

Nirmala X., Kodrík D., Žurovec M. \& Sehnal F. 2001a: Insect silk contains a Kunitz-type and a unique Kazal-type proteinase inhibitor. - Eur. J. Biochem. 268: 2064-2073.
Nirmala X., Mita K., Vanisree V., Žurovec M. \& Sehnal F. 2001b: Identification of four small molecular mass proteins in the silk of Bombyx mori. — Insect Mol. Biol. 10: 437-445.

Prudhomme J.C., Couble P., Garel J.P. \& Daillie J. 1985: Silk synthesis. In Kerkut G.A. \& Gilbert L.I. (eds): Comprehensive Insect Physiology, Biochemistry and Pharmacology. Vol. 10. Pergamon Press, Oxford, pp. 571-594.

Shaik H.A. \& Sehnal F. 2009: Hemolin expression in the silk glands of Galleria mellonella in response to bacterial challenge and prior to cell disintegration. - J. Insect Physiol. 55: 781-787.

Singh C.P., Vaishna R.L., Kakkar A., Arunkumar K.P. \& NAGARAJU J. 2014: Characterization of antiviral and antibacterial activity of Bombyx mori seroin protein. - Cell Microbiol. 16: 1354-1365.

Sutherland T.D., Young J.H., Weisman S., Hayashi C.Y. \& MERRITT D.J. 2010: Insect silk: One name, many materials. Annu. Rev. Entomol. 55: 171-188.

Tso I.M., Wu H.C. \& Hwang I.R. 2005: Giant wood spider Nephila pilipes alters silk protein in response to prey variation. $-J$. Exp. Biol. 208: 1053-1061.

Žurovec M., Kludkiewicz B., Fedic R., Sulitkova J., Mach V., Kucerova L. \& Sehnal F. 2013: Functional conservation and structural diversification of silk sericins in two moth species. - Biomacromolecules 14: 1859-1866.

Žurovec M., Yang C., Kodrík D. \& Sehnal F. 1998: Identification of a novel type of silk protein and regulation of its expression. - J. Biol. Chem. 273: 15423-15428.

Žurovec M. \& SehNal F. 2002: Unique molecular architecture of silk fibroin in the waxmoth, Galleria mellonella. - J. Biol. Chem. 277: 22639-22647.

Received June 20, 2016; revised and accepted December 12, 2016 Published online January 27, 2017 